Article

\title{
Dependencies for Determining the Thermal Conductivity of Moist Capillary-Porous Materials
}

\author{
Abdrahman Alsabry ${ }^{1}$, Beata Backiel-Brzozowska ${ }^{2, *}$ and Vadzim I. Nikitsin ${ }^{3}$ \\ 1 Faculty of Civil Engineering, Architecture and Environmental Engineering, Institute of Civil Engineering, \\ University of Zielona Gora, 65-417 Zielona Góra, Poland; a.alsabry@wp.pl \\ 2 Faculty of Civil Engineering and Environmental Sciences, Institute of Civil Engineering and Transport, \\ Bialystok University of Technology, 15-351 Bialystok, Poland \\ 3 Faculty of Civil Engineering, Brest State Technical University, 224017 Brest, Belarus; sniki@protonmail.com \\ * Correspondence: b.backiel@pb.edu.pl; Tel.: +48-517-177-553
}

Received: 25 May 2020; Accepted: 18 June 2020; Published: 20 June 2020

check for updates

\begin{abstract}
A method of determining the effective thermal conductivity of moist capillary-porous materials has been proposed, in which calculations are carried out while taking into account all components of the system (solid, liquid and gas) at once. The method makes it easy to take into account the way water is distributed in the pore space of the material, either as isolated inclusions (drops) or as a continuous component, depending on the moisture content of the material. In the analysis of heat transport in moist capillary-porous materials, the theory of generalized conductivity is used and the structure of moist material is modeled using an ordered geometric structure consisting of identical unit cells in the form of a cube. An equation is obtained for calculating the effective conductivity of capillary-porous materials with isolated and continuous liquid inclusions, with adiabatic and isothermal division of the unit cell. The proposed method is compared to the previously proposed method of determining the effective thermal conductivity of moist materials, in which the three-component system is gradually reduced to a binary system, showing disadvantages of this method compared to the currently proposed. It has been shown that the proposed formulas grant the possibility of a sufficiently accurate prediction of experimental results using the experimental results of the thermal conductivity of moist aerated concrete.
\end{abstract}

Keywords: thermal conductivity; porous structure modelling; capillary-porous materials

\section{Introduction}

Capillary-porous materials, commonly used in building envelope structures, are exposed to intense thermal and hydric influences during operation. It follows from the data published in a number of works (e.g., [1-3]) that as a result of these influences, the moisture of external wall materials $\psi$ may increase to a value several times exceeding the values adopted when designing a building. Increasing the moisture content of wall materials causes a decrease in their thermal insulation properties and other negative effects. Therefore, the thermal conductivity of materials should be determined taking into account real operational moisture designated with appropriate calculation or experimental methods when designing external walls.

The analysis of works [4-7] shows that the correct experimental assessment of the thermal conductivity of moist porous materials is very difficult, because the measurements obtained for the same material with the same average moisture content may differ significantly (in some cases even several times). First, this can be explained by the character of wetting the pore surface with liquid (partial or total), the character of moisture distribution in the volume of sample (even or uneven), the measurement method (stationary or non-stationary), etc. Therefore, to determine the thermal 
conductivity of moist materials engineering calculation methods based on mathematical modeling of the coupled heat and moisture transport process should be used and developed.

Literature presents numerous formulas for determining thermal conductivity of dry porous materials using methods developed over recent decades. Many of those formulas were considered in $[8,9]$. However, it is difficult to get the compliance of the calculated and experimental values of thermal conductivity for moist materials, which is shown by the results of works $[4,5,10]$ in which the Krisher method or its modification has been used. The formulas used in the abovementioned works contain empirical coefficients, the determination of which is often fraught with errors. In addition, only in [5] the nature of the distribution of moisture in the liquid phase in the material is considered, which can only partially wet the material skeleton by concentrating locally in the form of drops or completely wet the material skeleton, depend on the level of moisture. The transition from partial to total wetting of the skeletons is accompanied by a significant increase in the effective thermal conductivity of the material. Moreover, the effect of the resistance of the porous medium to vapor diffusion is not taken into account in a sufficient way when assessing the effect of vapor diffusion on heat transfer.

Thus, due to the complexity of the problem of coupled heat and mass transport in capillary-porous materials, modern science does not propose widely recognized methods for calculating the conductivity of moist building materials, the results of which can be sufficiently confirmed by experimental measurements. Therefore, this paper is another attempt to develop a simple calculation method enabling the determination of the thermal conductivity of capillary-porous materials, which can be used to solve various engineering problems (design of building partitions, assessment of existing buildings, etc.). The first attempt was to develop a calculation scheme presented in [11], proposed for determining the effective thermal conductivity of polyurethane foams. The dependencies presented in the current publication are the result of further work in the chosen direction, aimed at simplifying the calculation procedures and extending the possibility of their application to other materials with capillary-porous structure.

\section{Problem Description}

Wet capillary-porous materials can be considered as heterogeneous systems of three components, consisting of a solid skeleton (1), gas (2) and liquid (3). With the known structure of such a system, its thermal conductivity $\lambda(\mathrm{W} /(\mathrm{m} \cdot \mathrm{K}))$ is a function of the thermal conductivity of components $\lambda_{\mathrm{i}}$ and their volumetric concentrations $\mathrm{m}_{\mathrm{i}}\left(\mathrm{m}^{3} / \mathrm{m}^{3}\right)$; that is:

$$
\lambda=\mathrm{f}\left(\lambda_{\mathrm{i}} ; \mathrm{m}_{\mathrm{i}}\right), \mathrm{i}=1,2,3 .
$$

To determine this dependency, it is expedient to use well-founded and effective methods of the theory of generalized thermal conductivity, using geometric models of the material structure [5]. Two different approaches to solving this problem are possible. In the first approach, the method of gradual reduction of the multi-component system to subsequent binary systems is used, while in the other, thermal conductivities $\lambda_{\mathrm{i}}$ and volumetric concentrations of all components are taken into account simultaneously.

Let us consider the scheme of the first method for the determination of the effective thermal conductivity of a three-component system. During the first stage of calculations, one of the components is excluded from the system, for example component 3. Then a binary system is obtained, in this case consisting of components 1 and 2 , already with different concentration values $\mathrm{m}_{1}{ }^{\prime}$ and $\mathrm{m}_{2}{ }^{\prime}$ $\left(\mathrm{m}_{2}{ }^{\prime}=1-\mathrm{m}_{1}{ }^{\prime}\right)$, related to $\mathrm{m}_{1}$ and $\mathrm{m}_{2}$ by dependencies:

$$
\mathrm{m}_{1}^{\prime}=\frac{\mathrm{m}_{1}}{\mathrm{~m}_{1}+\mathrm{m}_{2}} ; \mathrm{m}_{2}^{\prime}=\frac{\mathrm{m}_{2}}{\mathrm{~m}_{1}+\mathrm{m}_{2}}
$$

Then, based on known formulas for binary systems, presented for example in $[4,5,8]$, the thermal conductivity $\lambda_{12}$ is determined. When choosing these formulas, the structural properties of the binary 
systems under consideration should be taken into account, due to the liquid and gas being distributed in the pore space of the material in the form of continuous components or isolated inclusions.

During the second stage, the third component with the thermal conductivity $\lambda_{3}$ and concentration $\mathrm{m}_{3}$, as well as the first binary system with the thermal conductivity $\lambda_{12}$ and concentration $m_{12}=m_{1}+m_{2}$ are considered to be the next binary system. The formulas for the binary system are repeated and the effective thermal conductivity of the three-component system is calculated.

In the case under discussion, the combination of component indices (the order in which components are taken into consideration) is $12 \& 3$. For a three-component system, two more combinations of indices are possible: $13 \& 2$ and $23 \& 1$. As emphasized in [5], the order in which the components are reduced to binary systems has an impact on the calculated value of the effective thermal conductivity of the considered material (system).

The above-mentioned disadvantage can be avoided by using another, more justified approach, which simultaneously takes into account the thermal conductivities and volume concentrations of all three components. However, to date, formulas for calculating the thermal conductivity of three-component systems, which would take into account the structural properties of wet capillary-porous materials, are not known. The work here provides a solution to this problem based on the methods of the theory of generalized conductivity.

\section{Models of the Geometric Structure of Binary Systems and Dependencies for Determining Their Thermal Conductivity}

Various methods of modeling the structure of capillary-porous building materials are used to analyze the heat transport process in such materials. In this work, a widely used method of transition to the unit cell has been used. In porous materials, you can always isolate binary systems with a continuous structure and isolated inclusions. For example, if in open-porous materials water moistens the entire surface of the solid skeleton, then together with the gas it forms a binary system having a structure with continuous, interpenetrating components. They are geometrically equivalent components. On the other hand, when the liquid only partially moistens the hard skeleton to form droplets, it can be considered to be a discontinuous component consisting of insulated inclusions in the continuous gas or solid component. Similar binary systems can also be identified in materials with closed pores, e.g., solid skeleton as a continuous component and gas filling closed pores as a discontinuous component consisting of isolated inclusions. The components of the binary system of such a structure are not equal geometrically.

If the material is isotropic, then its real structure can be adequately replaced by its model, consisting of ordered unit cells in the simplest cubic form [5,12-14]. The symmetry of the unit cell in two mutually perpendicular directions makes it possible to describe the heat transfer process in $1 / 2,1 / 4$ and $1 / 8$ parts of it. The eighth part of the unit cell having a structure with interpenetrating components (e.g., solid skeleton and gas) is illustrated in Figure 1a. If the solid skeleton (component 1) is concentrated in the cell bars, then the relative cross-sectional dimension of the bar $\mathrm{c}=\Delta / \mathrm{L}$ is related to the volumetric concentration of the considered component $\mathrm{m}_{1}$ by equation [15]:

$$
c=0.5+\sin \left[\frac{\arcsin \left(2 \cdot m_{1}-1\right)}{3}\right] .
$$

The volumetric concentration of gas (component 2) is determined by the formula:

$$
\mathrm{m}_{2}=\mathrm{P}=1-\mathrm{m}_{1} .
$$

When selecting a unit cell structure with isolated inclusions, it is sufficiently justified to use the cubic form of inclusions [8]. In this case, the unit cell will be in the form of a cube in a cube with symmetry in two mutually perpendicular directions. Figure $1 b$ shows the eighth part of the said unit cell. It is obvious that the volume concentration of inclusions $\mathrm{m}_{2}=(1 / \mathrm{L})^{3}$. 

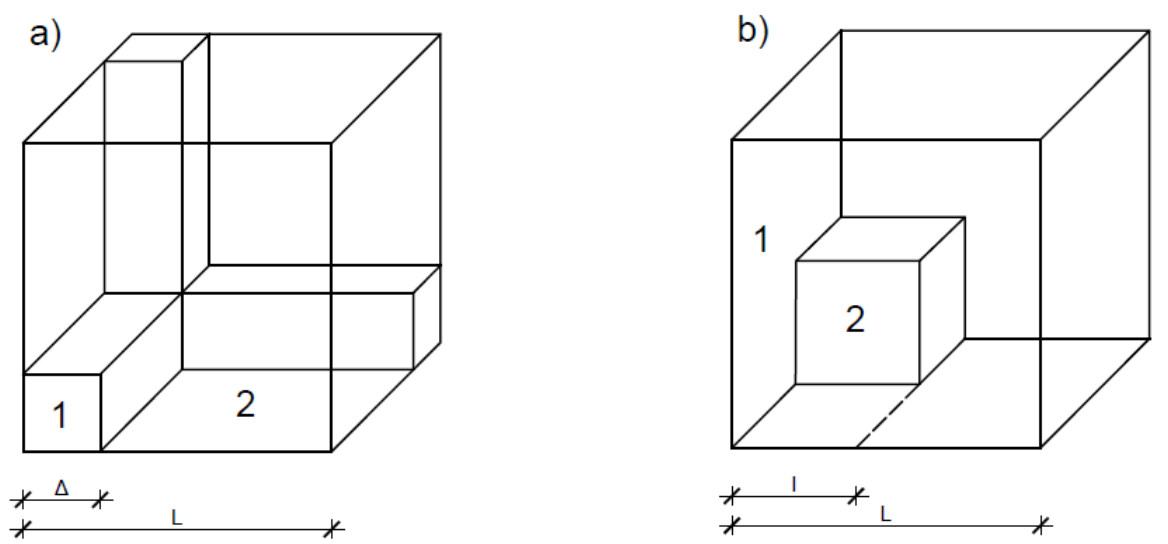

Figure 1. The eighth part of a cubic unit cell: (a) structure with two continuous, interpenetrating components (1 and 2); (b) structure with continuous component (1) and isolated cubic inclusions (2).

With the appearance of underhygroscopic liquid moisture with a volume concentration of $\mathrm{m}_{3}=\psi$, the volume concentration of the gas component $\mathrm{m}_{2}$ decreases and is equal to:

$$
\mathrm{m}_{2}=\mathrm{P}-\psi=1-\mathrm{m}_{1}-\mathrm{m}_{3}
$$

Let us consider the process of a gradual increase of moisture $\psi\left(\mathrm{m}^{3} / \mathrm{m}^{3}\right)$ in an open porous material and the accompanying structural changes that should be taken into account when calculating the effective thermal conductivity. At relatively low values of the material moisture $\psi$, the liquid will fill a small part of the pore space $\psi_{\mathrm{p}}=\psi / \mathrm{P}$ forming isolated inclusions (drops). As the pore moisture raises, the drops grow and when a certain boundary value $\psi_{\mathrm{p}}{ }^{\prime}$ is reached, the drops merge together and form a continuous inclusion. With a further increase of pore moisture to the next boundary value $\psi_{\mathrm{p}}{ }^{\prime \prime}$ the continuous distribution of gas changes into distribution in the form of isolated inclusions. Such a case is possible when the liquid is sucked by the material in contact with water due to capillary action. The boundary values of the pore moisture $\psi_{\mathrm{p}}{ }^{\prime}$ and $\psi_{\mathrm{p}}{ }^{\prime \prime}$ depend on the contact angle $\theta$ and the properties of the capillary-porous material structure. For the adopted hard skeleton model of the porous material (Figure 1a), the values of $\psi_{\mathrm{p}}{ }^{\prime}$ can be determined using the dependency $\psi_{\mathrm{p}}{ }^{\prime}=\mathrm{f}(\theta, \mathrm{c})$ presented in [11]. When deriving these dependencies, it is assumed that in the process of sorption moisture there is no capillary condensation and the maximum hygroscopic moisture of the material is very low $\left(\psi_{\mathrm{p}} \approx 0.002\right)$, and therefore it is not considered.

Let us determine formulas for calculating the pore moisture boundary values $\psi_{\mathrm{p}}$ " for a contact angle $\theta$ equal to 0,45 and $90^{\circ}$. The method of determining these dependencies for one value of the contact angle, for example $\theta=45^{\circ}$, will be considered. Figure 2 shows the eighth part of a unit cell with mutually penetrating components (solid skeleton and liquid) and isolated gas inclusions for the contact angle under consideration. Figure 2 shows that the volume of gas inclusions is equal to $\mathrm{V}_{\mathrm{g}}=1 / 6(\mathrm{~L}-\Delta)^{3}$, the pore volume $\mathrm{V}_{\mathrm{p}}=(\mathrm{L}-\Delta)^{3}+3 \Delta(\mathrm{L}-\Delta)^{2}=(\mathrm{L}-\Delta)^{2}(\mathrm{~L}+2 \Delta)$ and the liquid volume $\mathrm{V}_{\mathrm{w}}=\mathrm{V}_{\mathrm{p}}-\mathrm{V}_{\mathrm{g}}$. Then the boundary value of the pore moisture for $\theta=45^{\circ}$ is:

$$
\psi_{\mathrm{p}}^{\prime \prime}=\frac{\mathrm{V}_{\mathrm{w}}}{\mathrm{V}_{\mathrm{p}}}=\frac{5+13 \mathrm{c}}{6(1+2 \mathrm{c})}
$$

where $\mathrm{c}=\Delta / \mathrm{L}=\mathrm{f}\left(\mathrm{m}_{1}\right)$ is given by Equation (3).

Similarly, for $\theta=0^{\circ}$ we obtain the equation:

$$
\psi_{\mathrm{p}}^{\prime \prime}=\frac{6(1+2 \mathrm{c})-\pi(1-\mathrm{c})}{6(1+2 \mathrm{c})}
$$


and for $\theta=90^{\circ}$ :

$$
\psi_{\mathrm{p}}^{\prime \prime}=\frac{16+44 \mathrm{c}+\pi(1-\mathrm{c})}{20(1+2 \mathrm{c})}
$$

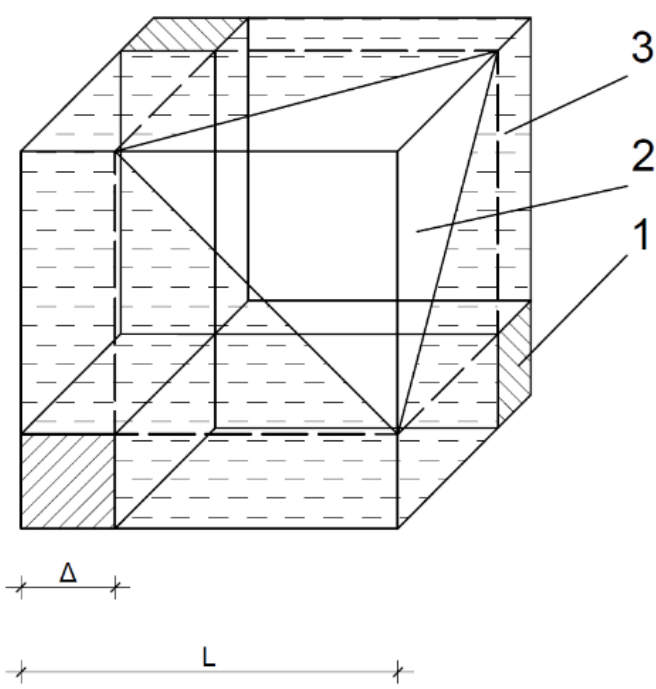

Figure 2. The eighth part of the unit cell at the boundary moisture $\psi_{\mathrm{p}}$ " and contact angle $\theta=45^{\circ}$; $1,2,3$ — solid, gas and liquid component of the structure, respectively.

An illustration of the dependency $\psi_{\mathrm{p}}{ }^{\prime \prime}=\mathrm{f}(\theta, \mathrm{c})$, determined using Equations (6)-(8), is shown in Figure 3. This figure also shows the dependency $\psi_{\mathrm{p}}{ }^{\prime}=\mathrm{f}(\theta, \mathrm{c})$ determined in [11]. These dependencies render it possible to assess the structure of the moist open-porous material as a three-component system, and then, using appropriate formulas, determine its effective thermal conductivity.

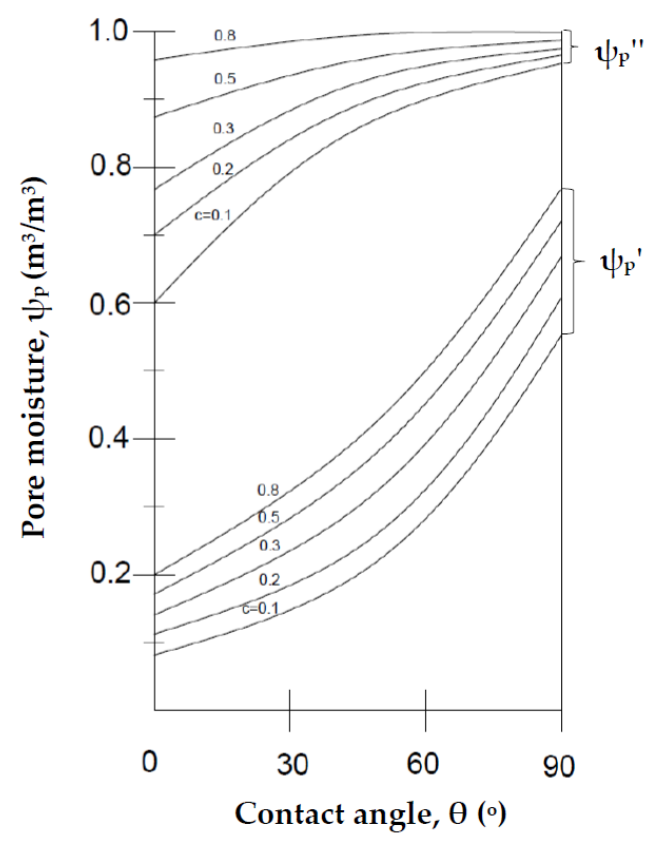

Figure 3. Dependence of the boundary pore moisture $\psi_{\mathrm{p}}{ }^{\prime}$ and $\psi_{\mathrm{p}}{ }^{\prime \prime}$ on the contact angle $\theta$ and relative dimension of the bar with a square cross section $\mathrm{c}=\Delta / \mathrm{L}=\mathrm{f}\left(\mathrm{m}_{1}\right)$.

When determining the effective thermal conductivity of three-component systems using the method of gradually reducing a multi-component system to a binary one various dependencies formulated for binary systems can be used. As part of the adopted theory of generalized thermal 
conductivity, the formulas for binary systems presented in [5] and determined by the division of unit cells shown in Figure 1 with planes parallel (adiabatic) and perpendicular (isothermal) to the heat flux can be used. Depending on the method of unit cells division, different formulas are obtained for determining the thermal conductivity. These equations will be presented, as they are needed for the subsequent comparative calculations.

The formulas for determining the thermal conductivity of a binary system with interpenetrating components have the form [5]:

- $\quad$ when dividing a unit cell (Figure 1a) with adiabatic planes:

$$
\frac{\lambda_{a}}{\lambda_{1}}=c^{2}+v(1-c)^{2}+2 v c(1-c)(v c+1-c)^{-1}
$$

where $v=\lambda_{2} / \lambda_{1}$

- $\quad$ when dividing this unit cell with isothermal planes:

$$
\frac{\lambda_{\mathrm{iz}}}{\lambda_{1}}=\left[\frac{1-\mathrm{c}}{\mathrm{c}^{2}+v\left(1-\mathrm{c}^{2}\right)}+\frac{\mathrm{c}}{\mathrm{c}(2-\mathrm{c})+v(1-\mathrm{c})^{2}}\right]^{-1} .
$$

The value of thermal conductivity at isothermal division $\lambda_{\mathrm{iz}}$ exceeds the value of thermal conductivity at adiabatic division $\lambda_{\mathrm{a}}$. The actual thermal conductivity $\lambda$ is between $\lambda_{\mathrm{iz}}>\lambda>\lambda_{\mathrm{a}}$ [5] Therefore, it is suggested to take the arithmetic mean as the final result:

$$
\lambda=\left(\lambda_{\mathrm{a}}+\lambda_{\mathrm{iz}}\right) / 2
$$

The thermal conductivity of the binary system with isolated inclusions, the structure of which is modeled by the unit cell shown in Figure 1b, is determined on the basis of the following Equations [5]:

- at adiabatic cell division

$$
\frac{\lambda_{\mathrm{a}}}{\lambda_{1}}=\frac{v-(v-1)\left(1-\mathrm{m}_{2}{ }^{\frac{2}{3}}\right) \mathrm{m}_{2}^{\frac{1}{3}}}{v-\mathrm{m}_{2}{ }^{\frac{1}{3}}(v-1)}
$$

- $\quad$ at isothermal cell division

$$
\frac{\lambda_{\mathrm{iz}}}{\lambda_{1}}=\frac{1+(v-1) \mathrm{m}_{2}{ }^{\frac{2}{3}}}{1+(v-1) \mathrm{m}_{2}{ }^{\frac{2}{3}}\left(1-\mathrm{m}_{2}{ }^{\frac{1}{3}}\right)} .
$$

Application of the presented dependencies formulated for binary systems to three-component systems does not allow for simultaneous consideration of all components in calculations. It is necessary to decide which two components are considered at the first stage of calculations, and which one to include at the second stage, while this order affects the final result. Therefore, it is necessary to formulate new dependencies that allow for calculation of thermal conductivity of moist capillary-porous materials while taking into account all components of the system at the same time. Such dependencies will be developed further in this work for both possible forms of liquid distribution (as an isolated inclusion and as a continuous component).

\section{Thermal Conductivity of Capillary-Porous Materials with Insulated Liquid Inclusions}

The structure of the moist material in the form of a unit cell with a drop of underhygroscopic liquid concentrated at its node is presented in Figure 1a. Depending on the value of the contact angle 
$\theta$, the surface of this drop of liquid can be both concave and convex [11]. As presented in [5] and confirmed by calculations made for the unit cell under consideration, the form (shape) of inclusion has a negligible effect on the effective thermal conductivity. Therefore, it is purposeful to use a cubic form of inclusion to make the analysis simple. In this case, the eighth part of the unit cell, as in [16], has the form shown in Figure 4. The dimension $x$ shown in Figure 4 is related to the volume of liquid $\mathrm{V}_{\mathrm{w}}$ by the formula $\mathrm{V}_{\mathrm{w}}=\mathrm{x}^{3}+3 \Delta \mathrm{x}^{2}$. By dividing both sides of this equation by the volume of the unit cell $\mathrm{V}=\mathrm{L}^{3}$, we get the cubic equation, associating the liquid volume concentration $\mathrm{m}_{3}=\psi$ (where $\psi$-material moisture) with the geometrical parameters $c=\Delta / L=f\left(m_{1}\right)$ i d $=x / L=f\left(m_{1}, m_{3}\right)$ :

$$
\mathrm{m}_{3}=\psi=\mathrm{d}^{3}+3 \mathrm{~cd} \mathrm{~d}^{2}
$$

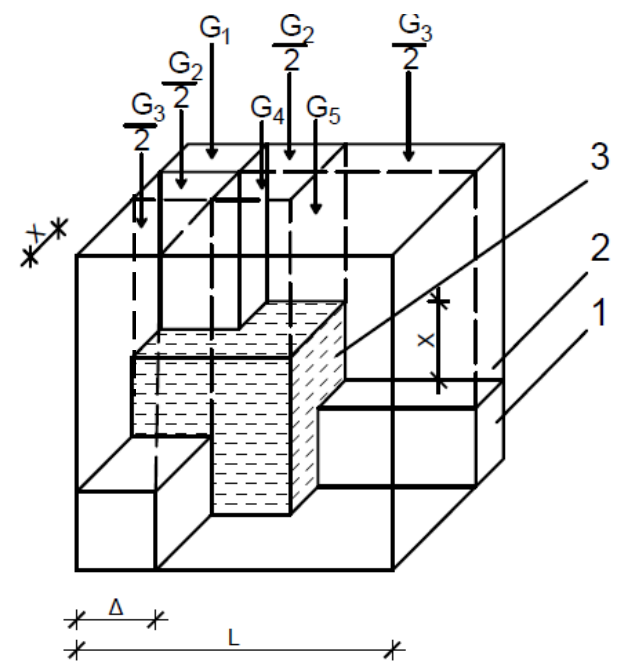

(a)

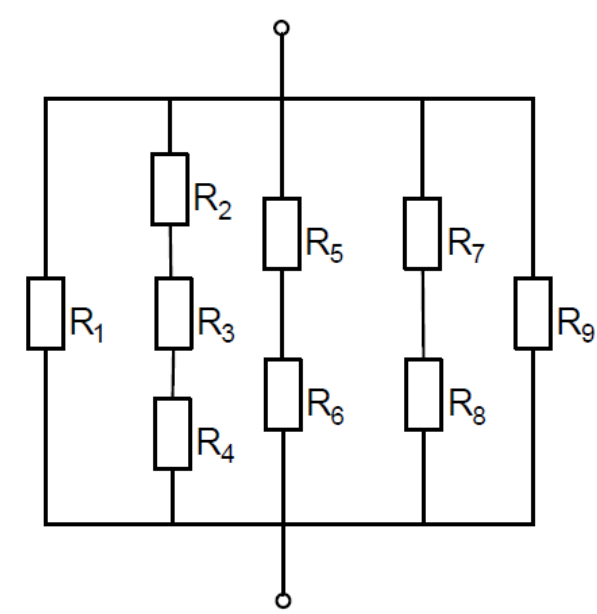

(b)

Figure 4. The eighth part of the unit cell with isolated liquid inclusion and $G_{1} \ldots G_{5}$ of the total heat flux $G$ (a) and a diagram of the connection of thermal resistances of the elements (b); 1, 2, 3-solid, gas and liquid component of the structure, respectively.

Once the values of $m_{3}$ and $c$ are known, the positive, physically justified root of parameter $d$ using Equation (14) is calculated.

Let us follow the method of determining the formula for calculating the effective thermal conductivity when dividing a unit cell (Figure 4a) with adiabatic planes (dashed lines) parallel to the lateral surface and the heat flux direction. As a result, the volume of the unit cell is divided into 14 elements, 9 of which are homogeneous. The thermal resistance of the i-th element of the unit cell is determined by the formula

$$
\mathrm{R}_{\mathrm{i}}=\frac{\mathrm{l}_{\mathrm{i}}}{\lambda_{\mathrm{i}} \cdot S_{\mathrm{i}}}
$$

where:

$\mathrm{l}_{\mathrm{i}}$-flux line length (height) of the i-th element [m],

$\lambda_{\mathrm{i}}$-thermal conductivity of the i-th element $[\mathrm{W} /(\mathrm{m} \cdot \mathrm{K})]$,

$\mathrm{S}_{\mathrm{i}}$-cross-sectional area of the $\mathrm{i}$-th element $\left[\mathrm{m}^{2}\right]$.

Let us assume that the general heat flow $G$ penetrating the unit cell consists of five interconnected fluxes $G_{1}, \ldots, G_{5}$, flowing through separate parts of the cell. The heat flux $G_{1}$ enters the hard skeleton 
bar, which has a length $l_{1}=L$, thermal conductivity $\lambda_{1}$ and a cross-sectional area $S_{1}=\Delta^{2}$. According to Equation (15), the resistance of a given element is:

$$
\mathrm{R}_{1}=\frac{\mathrm{L}}{\lambda_{1} \cdot \Delta^{2}}
$$

Flux $G_{2}$, with cross-section $S_{2}=2 \cdot x \cdot \Delta$, passes successively through gas, liquid and solid components, with resistances

$$
\mathrm{R}_{2}=\frac{\mathrm{L}-\Delta-\mathrm{x}}{\lambda_{2} \cdot 2 \cdot \mathrm{x} \cdot \Delta}, \mathrm{R}_{3}=\frac{\mathrm{x}}{\lambda_{3} \cdot 2 \cdot \mathrm{x} \cdot \Delta}, \mathrm{R}_{4}=\frac{\Delta}{\lambda_{1} \cdot 2 \cdot \mathrm{x} \cdot \Delta} .
$$

Flux $G_{3}$, with cross-section $S_{3}=2 \cdot \Delta \cdot(L-\Delta-x)$, passes through gas and solid components, whose resistances are as follows:

$$
\mathrm{R}_{5}=\frac{\mathrm{L}-\Delta}{\lambda_{2} \cdot 2 \cdot \Delta \cdot(\mathrm{L}-\Delta-\mathrm{x})}, \mathrm{R}_{6}=\frac{\Delta}{\lambda_{1} \cdot 2 \cdot \Delta \cdot(\mathrm{L}-\Delta-\mathrm{x})} .
$$

Flux $\mathrm{G}_{4}$, with cross section $\mathrm{S}_{4}=\mathrm{x}^{2}$, passes through gas and liquid components with resistances:

$$
\mathrm{R}_{7}=\frac{\mathrm{L}-\Delta-\mathrm{x}}{\lambda_{2} \cdot \mathrm{x}^{2}}, \mathrm{R}_{8}=\frac{\Delta+\mathrm{x}}{\lambda_{3} \cdot \mathrm{x}^{2}}
$$

Finally, flux $G_{5}$ with cross-section $S_{5}=(L-\Delta)^{2}-x^{2}$ passes through the gas component with resistance:

$$
\mathrm{R}_{9}=\frac{\mathrm{L}}{\lambda_{2} \cdot\left[(\mathrm{L}-\Delta)^{2}-\mathrm{x}^{2}\right]} .
$$

The scheme of connection of thermal resistances of all elements of the unit cell is shown in Figure $4 \mathrm{~b}$. In this case, the overall thermal resistance of the unit cell $\mathrm{R}$ can be calculated as follows:

$$
\frac{1}{\mathrm{R}}=\frac{1}{\mathrm{R}_{1}}+\frac{1}{\mathrm{R}_{2}+\mathrm{R}_{3}+\mathrm{R}_{4}}+\frac{1}{\mathrm{R}_{5}+\mathrm{R}_{6}}+\frac{1}{\mathrm{R}_{7}+\mathrm{R}_{8}}+\frac{1}{\mathrm{R}_{9}} .
$$

The overall thermal resistance of the analogous volume $\mathrm{V}=\mathrm{L}^{3}$, filled with a homogeneous substance with the effective thermal conductivity $\lambda_{a}$ is determined by the formula:

$$
\mathrm{R}=\frac{\mathrm{L}}{\lambda_{\mathrm{a}} \cdot \mathrm{L}^{2}}=\frac{1}{\lambda_{\mathrm{a}} \cdot \mathrm{L}}
$$

From Equation (21), taking into account Equations (16)-(20) and (22), after some transformations, we obtain the dependency for the effective thermal conductivity of a three-component system, one of which (liquid) is an isolated inclusion, when the cell is divided with adiabatic planes:

$$
\frac{\lambda_{a}}{\lambda_{1}}=c^{2}+\frac{2 \cdot v_{1} \cdot v_{2} \cdot c \cdot d}{v_{2} \cdot(1-c-d)+v_{1} \cdot v_{2} \cdot c+v_{1} \cdot d}+\frac{2 \cdot v_{1} \cdot c \cdot(1-c-d)}{v_{1} \cdot c+1-c}+\frac{v_{1} \cdot v_{2} \cdot d^{2}}{v_{2} \cdot(1-c-d)+v_{1} \cdot(c+d)}+v_{1} \cdot\left[(1-c)^{2}-d^{2}\right],
$$

where $v_{1}=\lambda_{2} / \lambda_{1}, v_{2}=\lambda_{3} / \lambda_{1}, c=\Delta / L$ and $d=x / L$.

In the absence of insulated inclusions of the liquid (dry material), parameters $v_{2}$ and $d$ are equal to zero and dependency (23) is transformed to Equation (9) determined by G. M. Dul'niev in 1965 [5].

In what follows, we determine the formula for calculating the effective thermal conductivity of the three-component system when dividing a unit cell with isothermal planes. The unit cell shown in Figure $4 \mathrm{a}$ will be divided with planes perpendicular to the direction of the general heat flux into three layers of different thickness. The thickness of the first (top) layer is equal to $h_{1}=L-\Delta-x$, the second (middle) layer $h_{2}=x$ and the third (bottom) layer $h_{3}=\Delta$. In each layer, each component is separated from the other by adiabatic planes parallel to the direction of the general heat flux. As a result, the 
first layer consists of two elements, and the last two layers of three elements, the thermal resistances of which can be determined using Equation (15). And so for the first layer, the following resistances are obtained:

$$
\mathrm{R}_{1}=\frac{\mathrm{L}-\Delta-\mathrm{x}}{\lambda_{1} \Delta^{2}}, \mathrm{R}_{2}=\frac{\mathrm{L}-\Delta-\mathrm{x}}{\lambda_{2}\left(\mathrm{~L}^{2}-\Delta^{2}\right)}
$$

For the second (middle) layer, the following equations are obtained:

$$
\mathrm{R}_{3}=\frac{\mathrm{x}}{\lambda_{1} \Delta^{2}}, \mathrm{R}_{4}=\frac{\mathrm{x}}{\lambda_{2}\left[\mathrm{~L}^{2}-(\Delta+\mathrm{x})^{2}\right]} ; \mathrm{R}_{5}=\frac{\mathrm{x}}{\lambda_{3}\left(2 \Delta \mathrm{x}+\mathrm{x}^{2}\right)} .
$$

For the third bottom layer, we have:

$$
\mathrm{R}_{6}=\frac{\Delta}{\lambda_{1}\left(2 \mathrm{~L} \Delta-\Delta^{2}\right)} ; \mathrm{R}_{7}=\frac{\Delta}{\lambda_{2}\left[(\mathrm{~L}-\Delta)^{2}-\mathrm{x}^{2}\right]} ; \mathrm{R}_{3}=\frac{\Delta}{\lambda_{3} \mathrm{x}^{2}} .
$$

The scheme of connection of thermal resistances of the three layers of the unit cell is shown in Figure 5.

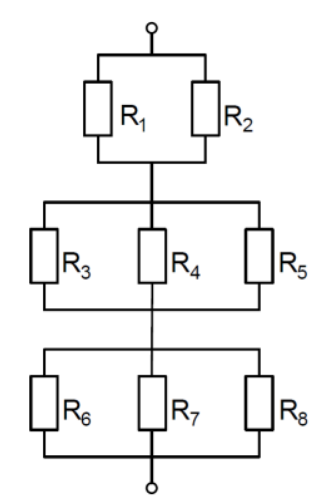

Figure 5. Diagram of connection of thermal resistances of separate elements at isothermal division.

For such a scheme, the overall thermal resistance of the unit cell $\mathrm{R}$ can be written as follows:

$$
\frac{1}{\mathrm{R}}=\left[\left(\frac{1}{\mathrm{R}_{1}}+\frac{1}{\mathrm{R}_{2}}\right)^{-1}+\left(\frac{1}{\mathrm{R}_{3}}+\frac{1}{\mathrm{R}_{4}}+\frac{1}{\mathrm{R}_{5}}\right)^{-1}+\left(\frac{1}{\mathrm{R}_{6}}+\frac{1}{\mathrm{R}_{7}}+\frac{1}{\mathrm{R}_{8}}\right)^{-1}\right]^{-1} .
$$

After substituting the resistances determined from Equations (24)-(26) to Equation (27) and taking into account Equation (22), we determine the dependency for calculating the effective thermal conductivity $\lambda_{z}$ of a three-component system when the unit cell is divided with isothermal planes shown in Figure 4a:

$$
\frac{\lambda_{\mathrm{iz}}}{\lambda_{1}}=\left[\frac{1-\mathrm{c}-\mathrm{d}}{\mathrm{c}^{2}+v_{1}\left(1-\mathrm{c}^{2}\right)}+\frac{\mathrm{d}}{\mathrm{c}^{2}+v_{1}\left[1-(\mathrm{c}+\mathrm{d})^{2}\right]+v_{2} \mathrm{~d}(2 \mathrm{c}+\mathrm{d})}+\frac{\mathrm{c}}{\mathrm{c}(2-\mathrm{c})+v_{1}\left[(1-\mathrm{c})^{2}-\mathrm{d}^{2}\right]+v_{2} \mathrm{~d}^{2}}\right]^{-1},
$$

where, as in Equation (23), $v_{1}=\lambda_{2} / \lambda_{1}, v_{2}=\lambda_{3} / \lambda_{1}, c=\Delta / L, d=x / L$. In the absence of a liquid component $\left(\lambda_{3}=0, m_{3}=0\right.$, then $\left.v_{2}=0, d=0\right)$ dependency (28) takes the form of Equation (10), given by Van Frey in 1935. The final value of the effective thermal conductivity $\lambda$ of an open porous material with insulated liquid inclusions is defined as the arithmetic mean of $\lambda_{\mathrm{a}}$ and $\lambda_{\mathrm{iz}}$, determined on the basis of (23) and (28).

Let us compare the results of calculations of the effective thermal conductivity of a three-component system obtained by the method of simultaneous consideration of all components and the method of gradual reduction of a multi-component system to a binary system. As an example, we will consider a 
system (moist material with open porosity) with known thermal conductivities and concentrations of three components: solid skeleton $\left(\lambda_{1}=1 \mathrm{~W} /(\mathrm{m} \cdot \mathrm{K})\right.$ and $\left.\mathrm{m}_{1}=0.5\right)$, gas $\left(\lambda_{2}=0.026 \mathrm{~W} /(\mathrm{m} \cdot \mathrm{K})\right.$ and $\left.\mathrm{m}_{2}=0.39\right)$ and liquid $\left(\lambda_{3}=0.6 \mathrm{~W} /(\mathrm{m} \cdot \mathrm{K})\right.$ and $\left.\mathrm{m}_{3}=0.11\right)$. Of course, the hard skeleton and gas are continuous and interpenetrating components. The character of the liquid inclusion will be explained, assuming a contact angle $\theta=45^{\circ}$. With known porosity $\mathrm{P}=1-\mathrm{m}_{1}=0.5$ and moisture of the material $\psi=\mathrm{m}_{3}=0.11$, the pore moisture $\psi_{\mathrm{p}}=\psi / \mathrm{P}=0.11 / 0.5=0.22$ is determined. Then from Equation (3), at $\mathrm{m}_{1}=0.5$ we determine $c=0.5$. Using the graphic dependency $\psi_{\mathrm{p}}{ }^{\prime}=f(\theta, c)$ in Figure 3 the boundary value of moisture content of the pores is found to be $\psi_{\mathrm{p}}{ }^{\prime} \approx 0.41$, which exceeds the actual $\psi_{\mathrm{p}}=0.22$. As assumed, the liquid in the material takes the form of isolated inclusions. Now, the thermal conductivity of the wet material will be calculated using both methods. The easiest way to do this is to take into account all components at the same time, using Equations (11), (23) and (28) and. Whereas, when calculating the thermal conductivity applying the method of gradual reduction of a multi-component system to a binary system, it is expedient to take into account all possible combinations of component indices, i.e.,: $12 \& 3,13 \& 2$ and $23 \& 1$. The scheme of implementation of this method for the combination of indices of components $12 \& 3$ has already been considered in the introduction, so it remains to specify this scheme taking into account that the liquid in the pore space may be in the form of isolated inclusions. In the first binary system with indices 12 (solid and gas phase), the components are uninterrupted and interpenetrate, therefore the thermal conductivity $\lambda_{12}$ is determined from Equations (9) and (10). In the next binary system, isolated liquid inclusions appear (index 3 ) and the calculation of the thermal conductivity is carried out using Equations (12) and (13). In the same way, calculations are made for other combinations of component indices. The results of calculations of the thermal conductivity of the three-component system under consideration based on both the methods described above are presented in Table 1.

Table 1. Calculated values of thermal conductivity of a porous material with isolated liquid inclusions (a three-component system) at adiabatic and isothermal division of the unit cell and their arithmetic means determined on the basis of the two methods used.

\begin{tabular}{ccccc}
\hline $\begin{array}{c}\text { The Method of Consideration } \\
\text { of Components }\end{array}$ & Gradual Reduction to Binary Structures & $\begin{array}{c}\text { Simultaneous } \\
\text { Consideration of } \\
\text { All Components }\end{array}$ \\
\hline Combination of Components Indices & $12 \& 3$ & $13 \& 2$ & $12 \& 3$ & 123 \\
$\lambda_{\mathrm{a}}$ & 0.3467 & 0.3527 & 0.2972 & 0.2948 \\
$\lambda_{\mathrm{iz}}$ & 0.4787 & 0.5031 & 0.4226 & 0.4729 \\
$\lambda=\left(\lambda_{\mathrm{a}}+\lambda_{\mathrm{iz}}\right) / 2$ & 0.4127 & 0.4279 & 0.3599 & 0.3838 \\
\hline
\end{tabular}

It follows from Table 1 that final results of calculations obtained by means of the method of gradual reduction to binary systems depend on the order in which components are considered. They may also significantly differ from the result obtained while considering all the components simultaneously. The indicated inaccuracies justify the need to simultaneously take into account all components when calculating the thermal conductivity of moist capillary-porous materials.

\section{Thermal Conductivity of Capillary-Porous Materials with Continuous Liquid Inclusion}

In this case, the open-porous material has a structure with three continuous components, which is presented in the form of a unit cell shown in Figure 6. It can be seen from the figure that underhygroscopic moisture wets the entire surface of the solid skeleton bars with a layer of constant thickness. The pore moisture cannot exceed the boundary value $\psi_{\mathrm{p}}$ " determined by Equations (6)-(8). 


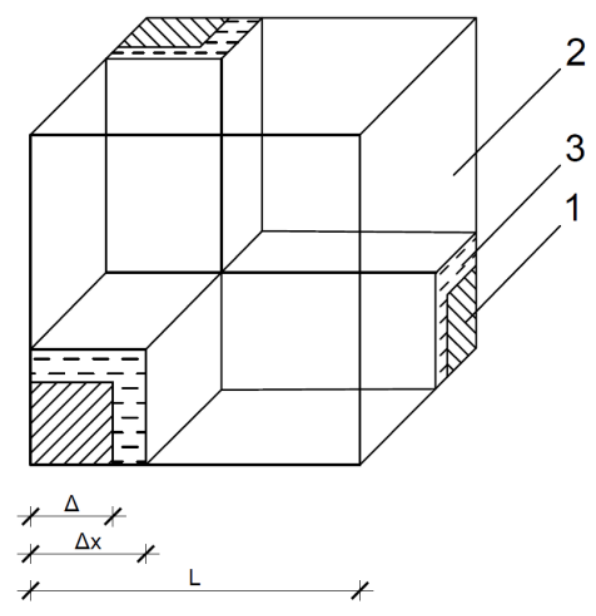

Figure 6. The eighth part of the unit cell with a continuous liquid inclusion.

After dividing the unit cell with adiabatic planes and carrying out the procedure used to derive Equation (23), a dependency is obtained for determining the effective thermal conductivity $\lambda_{a}$ of an open-porous material with a continuous liquid inclusion:

$$
\frac{\lambda_{a}}{\lambda_{1}}=c^{2}+\frac{2 v_{1} c\left(1-c_{x}\right)}{v_{1} c+1-c_{x}+\frac{v_{1}\left(c_{x}-c\right)}{v_{2}}}+\frac{2 v_{2} c\left(c_{x}-c\right)}{v_{2} c+1-c}+v_{1}\left(1-c_{x}\right)^{2}+v_{2}\left(c_{x}-c\right)^{2}+\frac{2 v_{1}\left(c_{x}-c\right)\left(1-c_{x}\right)}{1-c_{x}+v_{1} c_{x} / v_{2}},
$$

where $v_{1}=\lambda_{2} / \lambda_{1}, v_{2}=\lambda_{3} / \lambda_{1}, c=\Delta / L$ and $c_{x}=\Delta_{x} / L$. As before, the value of the $c$ parameter is determined from Equation (3). The value of the $c_{x}$ parameter is also determined from Equation (3), in which the sum of $m_{1}+m_{3}$ is used instead of $m_{1}$.

Division of the unit cell with isothermal planes leads to the following dependency for calculating the effective thermal conductivity $\lambda_{\mathrm{iz}}$ (upper assessment) of the capillary-porous material with a continuous liquid inclusion:

$$
\frac{\lambda_{\mathrm{iz}}}{\lambda_{1}}=\left[\frac{1-c_{\mathrm{x}}}{c^{2}+v_{1}\left(1-c^{2}\right)+v_{2}\left(c_{x}^{2}-c^{2}\right)}+\frac{c_{x}-c}{c^{2}+v_{1}\left(1-c_{x}\right)^{2}+v_{2}\left(2 c_{x}-c_{x}^{2}-c^{2}\right)}+\frac{c}{2 c-c^{2}+v_{1}\left(1-c_{x}\right)^{2}+v_{2}\left[2\left(c_{x}-c\right)-c_{x}^{2}+c^{2}\right]}\right]^{-1} ;
$$

in which the dimensionless parameters $v_{1}, v_{2}, c$ and $c_{x}$ are the same as in Equation (29). The final value of the thermal conductivity $\lambda$ is determined as the arithmetic mean $\lambda=\left(\lambda_{\mathrm{a}}+\lambda_{\mathrm{iz}}\right) / 2$.

To check whether Equations (29) and (30) can be used to predict experimental results the data obtained for aerated concrete are used [6]. It should be emphasized that for this material a continuous liquid inclusion can appear even if the material is not in contact with water. The maximum hygroscopic moisture $\psi_{\mathrm{h}}$ can reach 0.1 and more. Aerated concrete samples had an average apparent density of $485 \mathrm{~kg} / \mathrm{m}^{3}$ and porosity $\mathrm{P}=0.773$. Their thermal conductivity was measured at an average temperature of $\mathrm{t}=10^{\circ} \mathrm{C}$. The thermal conductivity of dry samples was $\lambda_{\text {dry }}=0.095 \mathrm{~W} /(\mathrm{m} \cdot \mathrm{K})$. The thermal conductivity of wet samples varied depending on the value of underhygroscopic moisture $\psi$. For example, at $\psi=0.185$, the measured thermal conductivity was $0.202 \mathrm{~W} /(\mathrm{m} \cdot \mathrm{K})$. For this case, demonstrative calculations are performed. Let us determine the thermal conductivity $\lambda_{\mathrm{i}}$ and volume concentration of components. The volume concentration of components is as follows: the solid skeleton $\mathrm{m}_{1}=1-$ $\mathrm{P}=0.227$, gas (vapor-air mixture) $\mathrm{m}_{2}=\mathrm{P}_{\psi}=0.588$ and water $\mathrm{m}_{3}=\psi=0.185$, whereas the thermal conductivity of water at $\mathrm{t}=10{ }^{\circ} \mathrm{C}$ is $\lambda_{3}=0.575 \mathrm{~W} /(\mathrm{m} \cdot \mathrm{K})$. The thermal conductivity of the vapor-gas mixture $\lambda_{2}$ is calculated as the sum of the thermal conductivity of dry air $\lambda_{\text {air }}=0.0249 \mathrm{~W} /(\mathrm{m} \cdot \mathrm{K})$ and the thermal conductivity caused by the diffusion of water vapor $\lambda_{\mathrm{V}}$, which was determined on the basis of the method described in [11] as $\lambda_{\mathrm{v}}=0.0066 \mathrm{~W} /(\mathrm{m} \cdot \mathrm{K})$. Then $\lambda_{2}=\lambda_{\text {air }}+\lambda_{\mathrm{v}}=0.0315 \mathrm{~W} /(\mathrm{m} \cdot \mathrm{K})$.

To determine $\lambda_{1}$ Equation (9) will be used, assuming $\lambda_{\mathrm{a}}=\lambda_{\mathrm{dry}}=0.095 \mathrm{~W} /(\mathrm{m} \cdot \mathrm{K})$. From Equation (3) at $\mathrm{m}_{1}$ the parameter $\mathrm{c}=0.3083$ is determined, writing $v=\lambda_{\text {air }} / \lambda_{1}$. As a result, in Formula (9) there is one unknown $\lambda_{1}$, which is determined by iteration (Newton's procedure), and we obtain 
$\lambda_{1}=0.715 \mathrm{~W} /(\mathrm{m} \cdot \mathrm{K})$. Next, Equations (29) and (30) are used and the lower $\lambda_{\mathrm{a}}=0.1646 \mathrm{~W} /(\mathrm{m} \cdot \mathrm{K})$ and the upper $\lambda_{\mathrm{iz}}=0.217 \mathrm{~W} /(\mathrm{m} \cdot \mathrm{K})$ assessments are calculated, the average of which gives the final calculation value $\lambda=\left(\lambda_{\mathrm{a}}+\lambda_{\mathrm{iz}}\right) / 2=0.191 \mathrm{~W} /(\mathrm{m} \cdot \mathrm{K})$, which turneds out to be smaller than the experimental one $\lambda_{\mathrm{w}}=0.202 \mathrm{~W} /(\mathrm{m} \cdot \mathrm{K})$ by $5.4 \%$.

The results of calculations of the thermal conductivity at other values of $\psi$ and experimental data are shown in Figure 7, from which it can be seen that the formulas obtained allow a sufficiently accurate prediction of experimental data. The shown convergence indicates the usefulness of the proposed dependencies for calculating the thermal conductivity of moist capillary-porous materials.

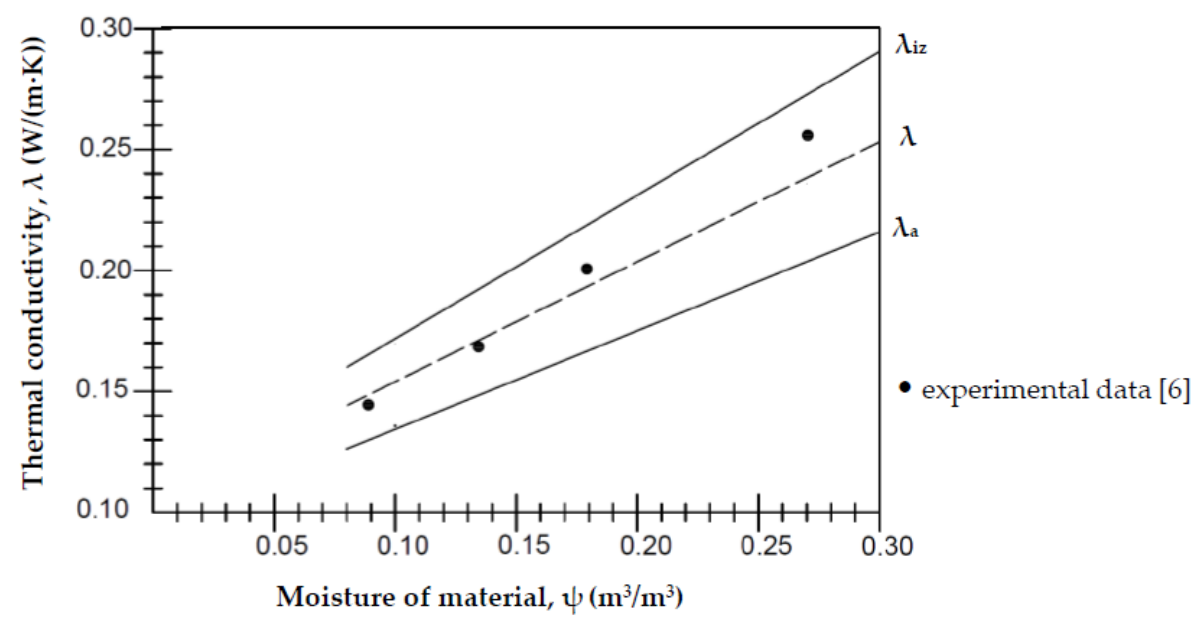

Figure 7. Calculated dependencies of thermal conductivity $\lambda_{\mathrm{a}}, \lambda_{\mathrm{iz}}$ and $\lambda=\left(\lambda_{\mathrm{a}}+\lambda_{\mathrm{iz}}\right) / 2$ on material moisture $\psi$ determined for aerated concrete with apparent density $\varrho=485 \mathrm{~kg} / \mathrm{m}^{3}$ and experimental data from work [6].

\section{Summary}

Two methods of determining the effective thermal conductivity of a moist capillary-porous material considered as a three-component system are compared. In the first method, the three-component system is gradually reduced to two successive binary systems, whereas in the other, all components are taken into account simultaneously. The disadvantages of the first method are demonstrated and compared to the other method, which is more reasonable. When analyzing heat transport in wet capillary-porous materials, the theory of generalized conductivity is used and the structure of a wet material is modeled using an ordered structure consisting of unit cells in the form of a cube. Through the approach adopted and taking into account all components simultaneously, a formula is obtained for calculating the effective conductivity of capillary-porous materials with isolated and continuous liquid inclusions at adiabatic and isothermal division of the unit cell. It is found that the results of calculations based on the formulas obtained predict experimental data sufficiently accurately.

The presented dependencies can be used to determine the thermal conductivity of various capillary-porous materials, in particular high-porous insulation and insulation-construction materials, such as polyurethane foam or aerated concrete. To perform calculations, only basic information about moist material is needed, such as porosity and moisture level. Calculations can be carried out for any moisture content of the material, both in hygroscopic and under-hygroscopic range, assuming any temperature. It can be helpful in solving various engineering problems, for example for building design and assessment of the technical conditions of operated buildings.

Further work on the presented methodology should aim at its calibration for the high level of material moisture, where the accuracy of experimental data prediction is slightly lower. 
Author Contributions: Conceptualization, V.I.N.; methodology, A.A.; software, B.B.-B.; validation, B.B.-B.; formal analysis, B.B.-B.; resources, A.A. and V.I.N.; data curation, A.A.; writing-Original draft preparation, V.I.N. and B.B.-B.; writing-Review and editing, B.B.-B. and A.A.; visualization, B.B.B; supervision, A.A.; funding acquisition, A.A. and B.B.-B. All authors have read and agreed to the published version of the manuscript.

Funding: This research is partly funded from the funds for education of the Ministry of Science and Higher Education in Poland, project number WZ/WB-IIL/3/2020 (Bialystok University of Technology).

Conflicts of Interest: The authors declare no conflict of interest.

\section{Nomenclature}

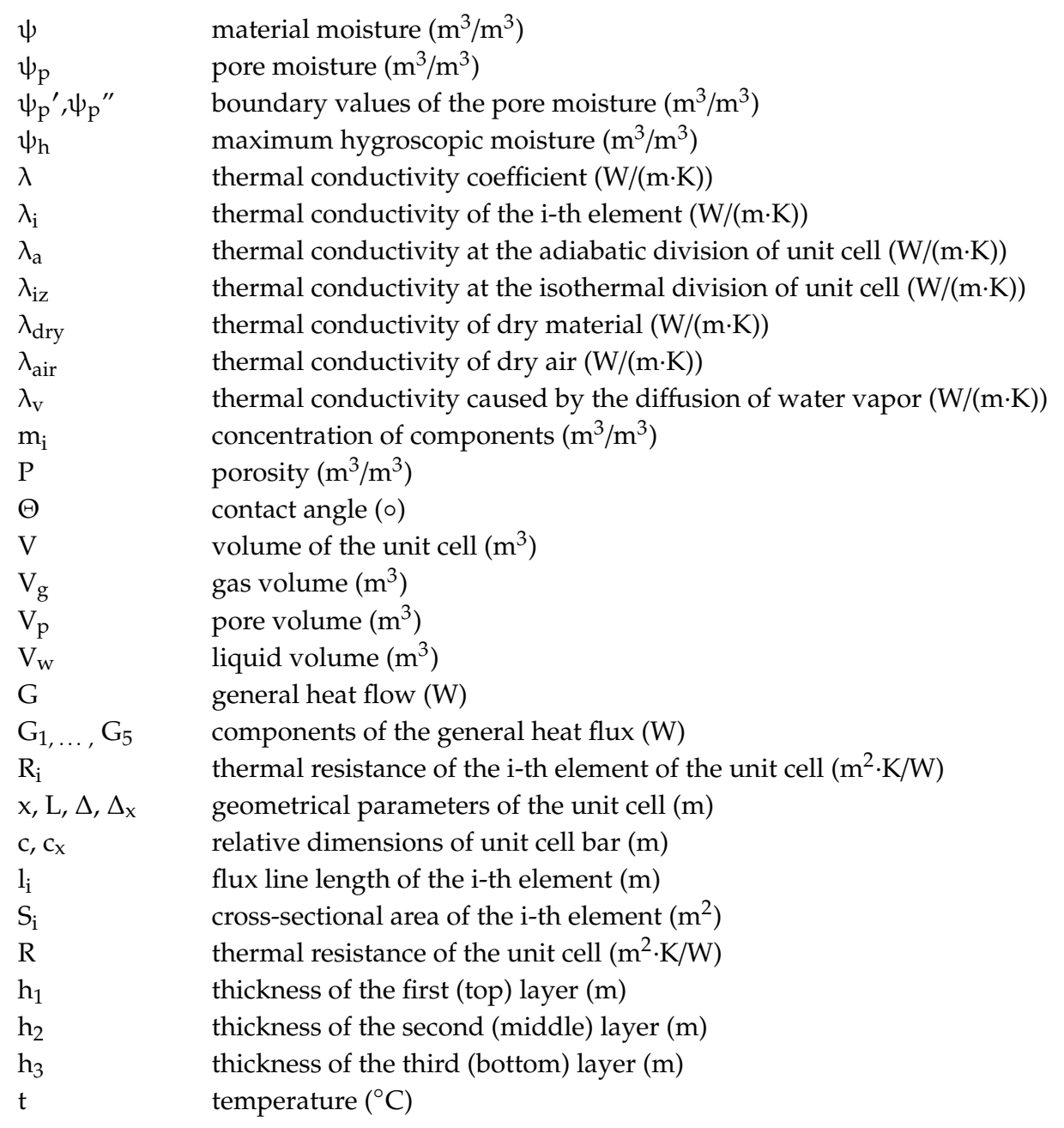

\section{References}

1. Blocken, B.; Carmeliet, J. A review of wind-driven rain research in building science. JWEIA 2004, 92, 1079-1130. [CrossRef]

2. Blocken, B.; Carmeliet, J. On the validity of the cosine projection in wind-driven rain calculations on buildings. Build Environ. 2006, 41, 1182-1189. [CrossRef]

3. Alsabry, A.; Nikitsin, V.I.; Kofanov, V.A.; Backiel-Brzozowska, B. Evaluation of the influence of wind-driven rain on moisture in cellular concrete wall boards. Int. J. Appl. Mech. Eng. 2017, 22, 509-519. [CrossRef]

4. Gur'ev, V.V.; Žoludov, V.S.; Petrov-Denisov, V.G. Teplovâ̂ Izolâciâ v Promyšlennosti. Teoriâ $i$ Rasčet; Strojizdat: Moscow, Russia, 2003.

5. Dul'nev, G.N.; Novikov, V.V. Processy Perenosa v Neodnorodnyh Sredah; Energoatomizdat: Leningrad, Russia, 1991.

6. Bobociński, A. Wpływ wilgotności ponadsorpcyjnej na przewodność cieplną betonów komórkowych. Build. Res. Inst. Q. 2004, 4, 3-12. 
7. Świńska, A.; Garbalińska, H. Thermal conductivity coefficient of cement-based mortars as air relative humidity function. Int. J. Heat Mass Transf. 2001, 47, 1077-1087. [CrossRef]

8. Collishaw, P.G.; Evans, J.R.G. An assessment of expressions for the apparent thermal conductivity of cellular materials. J. Mater. Sci. 1994, 29, 486-498. [CrossRef]

9. Alsabry, A.; Nikitsin, V.; Backiel-Brzozowska, B.; Kofanov, V. An analysis of relations for determining the thermal conductivity of ridge polymer foams. Int. J. Appl. Mech. Eng. 2018, 23, 1015-1023. [CrossRef]

10. Ochs, F.; Heidemann, W.; Müller-Steinhagen, H. Effective thermal conductivity of moisture insolation as a function of temperature. Int. J. Heat Mass Transf. 2008, 51, 539-552. [CrossRef]

11. Nikitsin, V.I.; Alsabry, A.; Kofanov, V.A.; Backiel-Brzozowska, B.; Truszkiewicz, P. A model of moist polymer foam and a scheme for the calculation of its thermal conductivity. Energies 2020, 13, 520. [CrossRef]

12. Glicksman, L.R. Heat transfer in foams. In Low Density Cellular Plastics; Hilyard, N.C., Cunningham, A., Eds.; Springer: Dordrecht, The Netherlands, 1994.

13. Coquaed, R.; Bailis, D. Numerical investigation of conductive heat transfer in high-porosity foams. Acta Mater. 2009, 57, 5466-5479. [CrossRef]

14. Mendes, M.A.A.; Ray, S.; Trimis, D. An improved model for effective thermal conductivity of open-cell porous foams. Int. J. Heat Mass Transf. 2011, 47, 1077-1087. [CrossRef]

15. Nikitsin, V.I.; Backiel-Brzozowska, B. Determination of capillary tortuosity coefficient in calculations of moisture transfer in building materials. Int. J. Heat Mass Transf. 2013, 56, 30-34. [CrossRef]

16. Dul'nev, G.N.; Volkov, D.P.; Malarev, V.I. Thermal conductivity of moist porous materials. J. Eng. Phys. 1989, 56, 198-206. [CrossRef]

(C) 2020 by the authors. Licensee MDPI, Basel, Switzerland. This article is an open access article distributed under the terms and conditions of the Creative Commons Attribution (CC BY) license (http://creativecommons.org/licenses/by/4.0/). 